\title{
Queima Inadequada de Resíduos Sólidos Domésticos, Principais Gases Tóxicos e Manifestações Clínicas: Uma Revisão de Literatura
}

\author{
Eutrópio Luiz Brandão Júniorl, Gabriel Pereira Bernardo², Lorena Pereira Bernardo ${ }^{3}$, \\ Sâmia Israele Braz do Nascimento ${ }^{4}$, Bruna Furtado Rolim Lima ${ }^{4}$, Kimberly Virginin Cruz Correia da Silva ${ }^{4}$, \\ Gabriela Macêdo Egídio Cavalcante ${ }^{4}$, Ana Laís Lacerda Rulim ${ }^{4}$, Jacob Oliveira Duarte ${ }^{5}$
}

Resumo: Na maioria dos municípios brasileiros, existem depósitos sem nenhum tipo de controle ou cuidado ambiental, chamados de vazadouros a céu aberto - os lixões. Neste cenário, a incineração irregular e as queimadas clandestinas dos resíduos sólidos domésticos produzem gases ricos em substâncias potencialmente tóxicas. Esta revisão buscou na literatura os principais gases tóxicos e suas manifestações clínicas associadas. Após a combustão dos resíduos domésticos, uma grande quantidade de gases e partículas sólidas são liberados na atmosfera, a exemplo do monóxido de carbono $(\mathrm{CO})$, metano $(\mathrm{CH} 4)$, hidrocarbonetos leves, compostos orgânicos voláteis (COV), orgânicos semi-voláteis (COSV), hidrocarbonetos aromáticos policíclicos (HAP), chumbo (Pb), mercúrio $(\mathrm{Hg})$, dibenzenodioxinas-policlorados (PCDDs), dibenzofuranos-policlorados (PCDFs) e os bifenilos-policlorados (PCBs). Tais componentes estão associados a algumas manifestações clínicas respiratórias, reprodutivas e oncogênicas com elevado impacto na saúde da população. Dessa forma, para entender os efeitos diretos e indiretos do destino inadequado dos resíduos sólidos e suas consequências é imprescindível mais investimentos e projetos para criação de aterros sanitários controlados e mais estudos relacionados à temática.

Palavras-chave: Queima. Gases Tóxicos. Resíduo Sólido Doméstico.

\section{Inadequate Burning of Household Solid Waste, Major Toxic Gases and Clinical Manifestations: A Literature Review}

\begin{abstract}
In most Brazilian municipalities, there are deposits without any kind of environmental control or care, called open pit dumps - the dumps. In this scenario, irregular incineration and clandestine burning of domestic solid waste produce gases that are rich in potentially toxic substances. This review sought in the literature the main toxic gases and their associated clinical manifestations. After combustion of domestic waste, a large amount of gases and solid particles are released into the atmosphere, such as carbon monoxide (CO), methane (CH4), light hydrocarbons, volatile organic compounds (VOCs), semi-volatile organic compounds (COSV), polycyclic aromatic hydrocarbons (HAP), lead $(\mathrm{Pb})$, mercury $(\mathrm{Hg})$, polycyclic dibenzenedioxins (PCDDs), dibenzofurans-
\end{abstract}

\footnotetext{
${ }^{1}$ Graduação em Medicina pela Faculdade de Medicina de Juazeiro do Norte, Brasil;

${ }^{2}$ Acadêmico de medicina Faculdade de Medicina Estácio de Juazeiro do Norte, Brasil.

Contato: gabrielpbernardo@ hotmail.com

${ }^{3}$ Graduada em Medicina pela Faculdade Estácio de Medicina do Juazeiro - FMJ e em Enfermagem pela Universidade Regional do Cariri- URCA, Pós-Graduada em Estratégia de Saúde da Família pela Universidade Vale do Acaraú (UVA) e em MBA Auditoria de Sistemas de Saúde pela Faculdade Estácio de Medicina do Juazeiro - FMJ. Contato: lorenapbernardo@hotmail.com

${ }^{4}$ Acadêmica de medicina na Faculdade de Medicina Estácio de Juazeiro do Norte, Brasil;

${ }^{5}$ Graduação em Medicina pela Universidade Federal do Ceará. Pós-graduação em Clínica Médica no Hospital Geral de Fortaleza. Pós-graduação em Pneumologia no Hospital Dr Carlos Alberto Studart Gomes - Hospital de Messejana. Pósgraduação em Broncoscopia no Hospital Dr Carlos Alberto Studart Gomes - Hospital de Messejana. Coordenador da Residência de Clínica Médica da Estácio/FMJ. Professor da Faculdade de Medicina Estácio de Juazeiro do Norte, Brasil.
} 
polychlorinated (PCDFs) and polychlorinated biphenyls (PCBs). These components are associated with some respiratory, reproductive and oncogenic clinical manifestations with a high impact on the health of the population. Thus, in order to understand the direct and indirect effects of the inadequate destination of solid waste and its consequences, more investments and projects for the creation of controlled landfills and more studies related to the subject are essential.

Keywords: Burning. Toxic Gases. Residuals

\section{Introdução}

O desenvolvimento acelerado, e por vezes desordenado dos municípios brasileiros acarretam problemas sociais gravíssimos, entre eles, a falta de políticas públicas destinadas ao descarte dos resíduos sólidos (SEIBERT, 2014).

No Brasil, as questões ambientais ocorrem paralelamente ao desenvolvimento da saúde coletiva, e tal desarticulação impossibilita experiências interdisciplinares que a complexa busca de soluções exige (PORTO, 1998). Nessa esfera, o próprio déficit de investimento na saúde pelo poder público repercute diretamente no setor específico de resíduos (DIAZ; SAVAGE; EGGERTH, 1997). Apesar da significativa melhora nas últimas duas décadas, os resíduos sólidos não apresentam destinação sanitária ambientalmente adequada, depositando - se em vazadouros a céu aberto, mais conhecido como lixões, presentes em pelo menos metade dos municípios brasileiros (GOUVEIA, 2012), ao passo que grande quantidade de aterros sanitários construídos está localizada nos grandes centros urbanos (IBGE, 2010).

Além do crescimento em absoluto, o lixo atual apresenta maior composição de materiais sintéticos e de maior periculosidade, em virtude da constante incorporação tecnológica (FERREIRA; ANTONIO, 2001). Os agentes químicos merecem destaque em decorrência da sua frequência, a exemplo das pilhas e baterias; óleos e graxas; pesticidas/herbicidas; solventes; tintas; produtos de limpeza; cosméticos; aerossóis e remédios. Outro aspecto importante é o acúmulo de resíduos biológicos que servem como proliferação de microrganismos, que são transmitidos de várias formas ao ser humano (FONSECA, 2009).

O descarte inadequado de inúmeros materiais, ocasionam complicações como a contaminação do solo, água e ar (GIUSTI, 2009), principalmente, através das dispersões ao 
solo, lixiviação, chorume e ar contaminado (FRANCHINI et al., 2004). Ademais, já está documentado em literatura inúmeras doenças associadas, que vão desde intoxicação por metais pesados, como maior incidência a vários tipos de cânceres (GOLDBERG; AL-HOMSI, 1995), problemas pediátricos e ginecológicos como o baixo peso ao nascimento, aborto e anomalias congênitas (FRANCHINI et al., 2004).

Uma das grandes e perigosas formas de intoxicação é a inalação de substâncias através da incineração ou queimada clandestina dos resíduos sólidos, produzindo grande quantidade de partículas ricas em metais pesados, compostos orgânicos e hidrocarbonetos que atingem de forma direta vários habitantes (WHO, 2007). Além disso, os profissionais que trabalham nessas áreas, que na sua grande maioria não fazem uso dos Equipamentos de Proteção Individual (EPI), podem desenvolver alterações na função pulmonar e infecções por contaminação do sistema respiratório por bactérias (KRAJEWSKI et al., 2002).

Com base nas evidências recentes, esta revisão da literatura pretende elencar e discutir as principais repercussões clínicas apresentadas nas populações expostas aos gases nocivos oriundos da queima inadequada dos resíduos sólidos.

\section{Materiais e Métodos}

O presente estudo é uma revisão de literatura conduzida a partir da seguinte pergunta norteadora: "Quais os gases tóxicos e suas principais manifestações clínicas decorrente da queima inadequada de resíduos sólidos?”. A revisão foi obtida via bases de dados eletrônicos: Literatura Latino-Americana e do Caribe em Ciências da Saúde (LILACS), Índice Bibliográfico Espanhol em Ciências da Saúde (IBECS) e Medical Literature Analysis and Retrieval System Online (MEDLINE). Os descritores utilizados foram: "burn" and "toxic gases" and "domestic solid waste".

Os critérios de inclusão selecionados foram: 1) artigos nos idiomas inglês, português e espanhol; 2) artigos que abordavam as manifestações clínicas relacionadas aos gases tóxicos liberados através da queima inadequada dos resíduos sólidos domésticos; 3) artigos pulicados no período entre 1990 e 2018. 
Foram encontrados 98 artigos, submetidos à análise do título e resumo após a busca inicial, resultando na seleção de 15 artigos (tabela 1), os quais foram lidos na íntegra e utilizados na construção deste artigo.

Tabela 1 - Lista de evidências encontradas na pesquisa.

\begin{tabular}{|c|c|c|c|}
\hline & Título & Ano & Autor \\
\hline 1 & $\begin{array}{l}\text { Case-Control Assessment of the Short-Term Health } \\
\text { Effects of an Industrial Toxic Waste Landfill }\end{array}$ & 1995 & DELORAINEA et al. \\
\hline 2 & $\begin{array}{l}\text { Risk of congenital anomalies near hazardous-waste } \\
\text { landfill sites in Europe: The EUROHAZCON study }\end{array}$ & 1998 & DOLK et al. \\
\hline 3 & Dioxinas e furanos: origens e riscos & 1999 & $\begin{array}{l}\text { ASSUNCAO; } \\
\text { PESQUERO }\end{array}$ \\
\hline 4 & $\begin{array}{l}\text { Environmental and Genetic Risk Factors and Gene- } \\
\text { Environment Interactions in the Pathogenesis of } \\
\text { Chronic Obstructive Lung Disease }\end{array}$ & 2000 & $\begin{array}{l}\text { WALTER; GOTTLIEB; } \\
\text { O'CONNOR }\end{array}$ \\
\hline 5 & $\begin{array}{c}\text { Incineração e dioxinas: Análise do aporte teórico } \\
\text { disponível }\end{array}$ & 2001 & $\begin{array}{l}\text { ALENCAR JÚNIOR; } \\
\text { GABAÍ }\end{array}$ \\
\hline 6 & Emissions of Organic Air Toxics from Open Burning & 2002 & US EPA \\
\hline 7 & Exposição aos agrotóxicos e câncer ambiental & 2003 & $\begin{array}{l}\text { KOIFMAN; } \\
\text { HATAGIMA }\end{array}$ \\
\hline 8 & $\begin{array}{l}\text { Effect of backyard burning on dioxin deposition and } \\
\text { air concentrations }\end{array}$ & 2004 & $\begin{array}{l}\text { WEVERS; DE FRÉ; } \\
\text { DESMEDT }\end{array}$ \\
\hline 9 & $\begin{array}{l}\text { Geração de energia elétrica a partir da incineração de } \\
\text { lixo urbano: o caso de Campo Grande/MS }\end{array}$ & 2005 & CAIXETA \\
\hline 10 & $\begin{array}{l}\text { Formation of toxic chemicals including dioxin- } \\
\text { related compounds by combustion from a small } \\
\text { home waste incinerator }\end{array}$ & 2006 & NAKAO et al. \\
\hline 11 & $\begin{array}{l}\text { Relatório da Qualidade do Ar da cidade de São } \\
\text { Paulo: } 2007\end{array}$ & 2007 & CETESB \\
\hline 12 & $\begin{array}{l}\text { Níveis ambientais e biológicos de dioxinas e furanos } \\
\text { em Portugal }\end{array}$ & 2008 & COUTINHO et al. \\
\hline 13 & Children are not little adults & 2008 & WHO \\
\hline 14 & $\begin{array}{l}\text { Air pollution and effects on reproductive-system } \\
\text { functions globally with particular emphasis on the } \\
\text { brazilian population }\end{array}$ & 2010 & VERAS et al. \\
\hline 15 & Neurobehavioural effects of developmental toxicity & 2014 & $\begin{array}{l}\text { GRANDJEAN; } \\
\text { LANDRIGAN }\end{array}$ \\
\hline
\end{tabular}

Fonte: Autoria própria, 2018. 


\section{Resultados e Discussão}

A queima dos resíduos em condições de combustão inferiores às ideais, produz partículas e gases nocivos chamados tipicamente de "cortina de fumaça", destacando-se o monóxido de carbono (CO), metano ( $\mathrm{CH} 4)$ e outros hidrocarbonetos leves, compostos orgânicos voláteis (COV), como benzeno, e compostos orgânicos semi-voláteis (COSV), incluindo hidrocarbonetos aromáticos policíclicos (HAP). Dependendo da fonte, quantidades variáveis de metais, como chumbo $(\mathrm{Pb})$ ou mercúrio $(\mathrm{Hg})$, podem ser emitidos, assim como dibenzenodioxinas-policlorados (PCDDs) e dibenzofuranos-policlorados (PCDFs) ou bifenilos-policlorados (PCBs) (US EPA, 2002).

A presença de compostos químicos varia de acordo com o tipo de lixo e a classe dos compostos químicos pesquisados no ar. Em um estudo analisado, os compostos orgânicos voláteis (COV) e hidrocarbonetos aromáticos policíclicos (HAP) estavam presentes em muitas fontes avaliadas, assim como os compostos orgânicos parcialmente voláteis não hidrocarboneto aromático policíclico; o que não foi verificado para os PCDDs e os PCDFs, presentes apenas em algumas pesquisas, pois tais emissões de PCDD e PCDF exibiram variações significativas nas categorias de origem dos resíduos. Esta grande variação é devido a uma combinação de fatores, incluindo composição de combustível, valor de aquecimento de combustível, densidade dos materiais, concentração de oxigênio e condições de combustão (ALENCAR JÚNIOR; GABAÍ, 2001).

Cinzas residuais da combustão de lixo doméstico foram analisadas usando um incinerador de pequeno porte a partir de materiais mistos contendo proporções variáveis de papeis (maior porção), fibras, plásticos não clorados, clorados e fios elétricos, plásticos clorados aumentaram a liberação de PCDD e PCDFs tanto no gás de combustão quanto na cinza. O fio de cobre também aumentou o valor de PCDD / PCDFs lançados, assim como seus precursores, como policlorados benzenos, fenóis policlorados, difenil policlorado éteres e bifenilos policlorados (NAKAO et al., 2006), visto também através da queima de resíduos em "barril de quintal" levando a emissão de PCDD e PCDF (WEVERS; DE FRÉ; DESMEDT, 2004).

A queima irregular de lixo doméstico é um dos principais contribuintes para liberação de gases tóxicos, sendo uma das maiores fontes geradoras de PCDDs / PCDFs (US EPA, 1994). Ademais, a queima dos resíduos sólidos produz grande quantidade de gás carbônico (CO2), 
óxido de enxofre (SOX), óxido de nitrogênio (NOX), nitrogênio (N2) e material particulado. São encontrados ácidos clorídrico $(\mathrm{HCl})$ e fluorídrico $(\mathrm{HF})$ em pequenas concentrações. Associados à combustão incompleta, há ainda a produção de monóxido de carbono (CO), hidrocarbonetos e, associados ao material particulado, tem-se a emissão de metais pesados (CAIXETA, 2005).

Coutinho et al. (2008) acompanharam durante cinco anos os níveis de PCDD e PCDF, de acordo com a sazonalidade, nas principais cidades de Portugal, constatando níveis maiores destas toxinas durante o inverno (Outubro a Março), evidenciando a existência de uma sazonalidade na variação dos níveis atmosféricos de dioxinas e furanos.

A poluição atmosférica causa grande impacto na saúde da população mundial, se tornando um importante fator de risco para várias doenças, como as de etiologia respiratória de evolução aguda e crônica, com destaque para asma, bronquite crônica e câncer de pulmão, levando direta e indiretamente a cerca de 2 a 4 milhões de mortes por ano (ASSUNÇÃO; PESQUERO, 1999).

Crianças em contato com gases tóxicos podem comprometer sua função pulmonar ou exacerbar condições pré-existentes aumentando a incidência de infecções respiratórias agudas, número de internações hospitalares e absenteísmo escolar. (WHO, 2008).

Ademais, crianças moradoras nas áreas de aterro sanitário apresentaram 30\% mais tosse ou sibilância assim como sintomas respiratórios inespecíficos, sendo a incidência diretamente proporcional ao tempo de exposição (DELORAINEA et al., 1995). Os efeitos dos gases são gradativos, como a tosse seca, cansaço, ardor nos olhos, nariz, garganta, além de desencadear aumento dos sintomas e agravamento das doenças respiratórias e cardiovasculares preexistentes (CETESB, 2007).

Vapores de metais presentes em nuvens tóxicas estão associados à Doença Pulmonar Obstrutiva Crônica (DPOC), relacionando o tempo de exposição à perda da função pulmonar, assim como a presença de outras substâncias irritantes como $\mathrm{Cl}, \mathrm{SO} 2, \mathrm{H} 2 \mathrm{~S}$, estireno, metileno, metacrilato entre outros. (WALTER; GOTTLIEB; O’CONNOR, 2000).

Estudos descreveram efeitos nocivos ao sistema reprodutor como alterações da fertilidade e saúde fetal, associando a exposição aos poluentes atmosféricos com baixo peso ao nascimento, retardo de crescimento intrauterino, prematuridade, morte neonatal, além da redução da fertilidade masculina e feminina (VERAS et al., 2010), aumento dos defeitos 
genéticos no sistema nervoso central e cardiopatias congênitas nas populações próximas a aterros em diversas regiões europeias (DOLK et al., 1998).

Produtos contidos em gases tóxicos, como as PCBs, Chumbo, Manganês e Mercúrio levam a neurotoxicidade através do contato direto, da inalação de gases ou da absorção pela pele, levando a alterações em processos de migração, diferenciação de sinapses, mielinização e apoptose. Nesse contexto, podem-se desenvolver distúrbios clínicos como déficit cognitivo, retardo mental, autismo, paralisia entre outros (GRANDJEAN; LANDRIGAN, 2014).

O 2,3,7,8-tetraclorodibenzo-p-dioxina (TCDD) age como promotor estimulando células de forma reversível induzindo à apoptose. Estudos adicionais afirmaram que o TCDD não apresenta um efeito carcinogênico completo, necessitando de certas condições de exposição para aumentar a incidência de câncer em humanos (ASSUNÇÃO; PESQUERO, 1999).

Diante da perspectiva de poucos estudos com significância estatística relevante associada aos TCDDs e a carcinogênese em seres humanos, as reduções dos níveis de TCDDs em última análise minimizam o impacto na saúde pública e ambiental (KOIFMAN; HATAGIMA, 2003). Em contraponto, apesar das dioxinas e furanos estarem no ar, água, solo, sedimentos, tecidos animais e vegetais, os teores observados são muito baixos e não existem evidências inequívocas de que o ser humano seja levado à morte ou tenha contraído câncer em função desses níveis habituais.

Há uma crença generalizada de que as concentrações de PCDD's e PCDF’s existentes no meio ambiente são toleráveis à vida humana. Não foram observadas alterações cromossômicas, nem teratogênicas em fetos de mulheres grávidas expostas, assim como em crianças e em adultos do sexo masculino, não demonstrando risco em desenvolver câncer (BROWNER, 1995).

De forma mais específica, os gases tóxicos relacionam-se a sinais e sintomas dosedependentes como relatados na tabela 2, segundo Silva (2004).

Tabela 2 - Sintomas associados à exposição individual de gases

\begin{tabular}{|c|c|}
\hline COMPOSTO & SINTOMAS \\
\hline $\begin{array}{c}\text { Monóxido de } \\
\text { carbono }\end{array}$ & $\begin{array}{c}\text { Ligeira cefaleia, cefaleia intensa, vertigem, taquicardia, } \\
\text { taquipneia, síncope, depressão respiratória e morte. }\end{array}$ \\
\hline $\begin{array}{c}\text { Óxidos de } \\
\text { Nitrogênio }\end{array}$ & $\begin{array}{c}\text { Asma, acessos de tosse com expectoração espumosa, edema } \\
\text { agudo de pulmão, bronquite, broncopneumonia, pulso irregular, }\end{array}$ \\
\hline
\end{tabular}




\begin{tabular}{|c|c|}
\hline & $\begin{array}{c}\text { coagulopatia, cianose, sudorese, sede intensa, morte por colapso } \\
\text { cardiovascular. }\end{array}$ \\
\hline $\begin{array}{l}\text { Dióxido de } \\
\text { Enxofre }\end{array}$ & Bronquite, asma e morte \\
\hline Ozônio & $\begin{array}{l}\text { Irritação nasal, diminuição da acuidade visual, cefaleia, dispneia, } \\
\text { tosse, distúrbios neurológicos, edema pulmonar, predisposição a } \\
\text { infecções e morte }\end{array}$ \\
\hline $\begin{array}{l}\text { Sulfeto de } \\
\text { Hidrogênio }\end{array}$ & $\begin{array}{l}\text { Conjuntivite dolorosa, cefaleia náuseas, tosse, tontura, sonolência } \\
\text { e edema pulmonar, convulsão, e morte por paralisia respiratória e } \\
\text { cardiovascular. Intoxicação crônica: queda da pressão arterial, } \\
\text { náuseas, perda de peso, distúrbios da marcha, tosse crônica }\end{array}$ \\
\hline Cloro & Tosse, dispneia, hemoptise, edema pulmonar, asfixia e morte \\
\hline
\end{tabular}

Fonte: SILVA (2004).

\section{Conclusões}

A incineração, como uma das opções para destinação de resíduos sólidos, vem aumentando em muitos países. Isso parece estar comprometendo a segurança da população que estão no entorno, onde acontecem tais queimadas, por conta dos efluentes que culminam sendo lançados ao ar. É preciso que as autoridades envolvidas possam discutir e avaliar sobre as tecnologias disponíveis para um adequado monitoramento de tais emissões, observando-se os possíveis efeitos na saúde das populações afetadas.

Apesar das diversas formas de armazenamento regular do lixo urbano, os investimentos destinados ao descarte de resíduos sólidos no Brasil estão abaixo do esperado, principalmente, nas Regiões Norte e Nordeste.

Ante o exposto, a queima inadequada parece ter sido a solução mais utilizada para darse destino aos resíduos sólidos no país. Dessa forma, gases tóxicos como monóxido de carbono, óxidos de nitrogênio, dióxido de enxofre, ozônio, sulfeto de hidrogênio e cloro, parecem ter um impacto negativo na população a tal situação esteja exposta.

Parece imprescindível a busca por minimizar a quantidade de resíduos desprezados pela sociedade. Há que se pensar em alternativas mais sustentáveis como a reutilização ou a reciclagem, seguindo-se um raciocínio mais ecológico. Tanto a redução como a reutilização e a reciclagem, podem ser incentivadas diretamente, por meio de ações educativas para atitudes de consumo e descarte mais conscientes por parte da população. Nem sempre é fácil ante as 
forças do mercado consumidor. Mas promover discussões e incentivar formas de produção e consumo ambientalmente sustentáveis, podem ajudar em mudanças comportamentais mais saudáveis.

É possível concluir que a poluição atmosférica associada à queima de resíduos sólidos domésticos apresenta repercussão à saúde humana, com predomínio de manifestações clínicas associadas ao aparelho respiratório, reprodutivo e os efeitos carcinogênicos, sobretudo nas populações menores de 18 anos e naquelas com múltiplas comorbidades.

As decisões que sobre como gerenciar os resíduos sólidos urbanos, são importantes mecanismos de saúde pública. Requerem firmes decisões políticas e econômicas pois, tem diretas consequências sociais e ambientais. Tais decisões, ao que nos parece, ainda representam um desafio, dada a sua natureza complexa, principalmente nas grandes cidades. É muito urgente e necessário que os gestores públicos objetivem ações preventivas para eliminação dos riscos à saúde e ao ambiente. É possível que tais medidas envolvam também, alguma garantia de inclusão social, de forma a permitir maior acesso a educação e, a uma alimentação de qualidade, mecanismos mitigadores de mudanças comportamentais e sanitárias mais saudáveis. Em uma perspectiva socialmente mais justa bem como ambientalmente mais sustentável, torna-se mais razoável discutir-se sobre desenvolvimento.

\section{Referências}

ALENCAR JÚNIOR, N. R., GABAÍ, I. Incineração e dioxinas: Análise do aporte teórico disponível. Encontro Nacional de Engenharia de Produção. Salvador, BA, 2001.

ASSUNCAO, J. V.; PESQUERO, C. R. Dioxinas e furanos: origens e riscos. Rev. Saúde Pública, São Paulo, v. 33, n. 5, p. 523-530, Out. 1999.

BROWNER, C. M. The epa's environmental justice strategy. 1995. Disponível em: < https://www.epa.gov/sites/production/files/2015-02/documents/ej_strategy_1995.pdf >. Acesso em: 12 out. 2017.

CAIXETA, D. M. Geração de energia elétrica a partir da incineração de lixo urbano: o caso de Campo Grande/MS. Brasília: Universidade de Brasília, Centro de desenvolvimento sustentável, 2005.

CETESB. Relatório da Qualidade do Ar da cidade de São Paulo: 2007. São Paulo, 2007. 
COUTINHO, M. et al. Níveis ambientais e biológicos de dioxinas e furanos em Portugal. 1998. (Instituto do Ambiente e Desenvolvimento, Campus Universitário, AVEIRO Departamento de Ambiente e Ordenamento). Disponível em: < https://www.ua.pt/idad/ readobject. aspx?obj=9475 > Acesso em: 15 ago. 2018.

DELORAINEA, A. et al. Case-Control Assessment of the Short-Term Health Effects of an Industrial Toxic Waste Landfill. Environmental Research, v. 68, n. 2, p. 124-132, 1995.

DIAZ, L. F.; SAVAGE, G. M.; EGGERTH, L. L. Managing solid wastes in developing countries. Wastes Management, v. 10, p. 43-45, 1997.

DOLK, H. et al. Risk of congenital anomalies near hazardous-waste landfill sites in Europe: The EUROHAZCON study. Lancet, v. 352, n. 9126, p. 423-427, 1998.

FERREIRA, J. A.; ANTONIO, L. Aspectos de saúde coletiva e ocupacional associados à gestão dos resíduos sólidos municipais Public and occupational health issues related to municipal solid waste management. Cad. Saúde Pública, v. 17, n. 3, p. 689-696, 2001.

FONSECA, J. C. L. Manual para Gerenciamento de Resíduos Perigosos, 2009.

FRANCHINI, M. et al. Health effects of exposure to waste incinerator emissions:a review of epidemiological studies. Annali dell'Istituto superiore di sanità, v. 40, n. 1, p. 101-15, 2004.

GIUSTI, L. A review of waste management practices and their impact on human health. Waste Management, v. 29, n. 8, p. 2227-2239, 2009.

GOLDBERG, M.; AL-HOMSI, N. Incidence of cancer among persons living near a municipal solid waste landfill site in Montreal, QuebecArchives of Environmental Health, 1995.

GOUVEIA, N. Resíduos sólidos urbanos: impactos socioambientais e perspectiva de manejo sustentável com inclusão social. Ciência \& Saúde Coletiva, v. 17, n. 6, p. 1503-1510, 2012.

GRANDJEAN, P.; LANDRIGAN, P. J. Neurobehavioural effects of developmental toxicity. Lancet, v. 13, n. 3, p. 330-338, 2014.

INTITUTO BRASILEIRO DE GEOGRAFIA E ESTATÍSTICA - IBGE. Diretoria de Pesquisa. Pesquisa Nacional de Saneamento Básico 2008. Rio de Janeiro, 2010.

KOIFMAN, S.; HATAGIMA, A. Exposição aos agrotóxicos e câncer ambiental. É Veneno Ou É Remédio, p. 75-100, 2003.

KRAJEWSKI, J. A et al. Occupational exposure to organic dust associated with municipal waste collection and management. International journal of occupational medicine and environmental health, v. 15, n. 3, p. 289-301, 2002.

NAKAO, T. et al. Formation of toxic chemicals including dioxin-related compounds by combustion from a small home waste incinerator. Chemosphere, v. 62, n. 3, p. 459-468, 2006. 
SEIBERT, A. L. A. Importância da gestão de resíduos sólidos urbanos e a conscientização sobre a sustentabilidade para a população em geral. 2014. Monografia (Especialização em Gestão Ambiental) - Universidade Tecnológico Federal do Paraná: Diretoria de Pesquisa e Pós-Graduação.

SILVA, L. C. Gases tóxicos em unidades armazenadoras. Grãos Brasil: da semente ao consumo, v. 3, n. 13, p. 12-15, mar. 2004.

PORTO, M. F. S. Saúde, ambiente e desenvolvimento: reflexões sobre a experiência da COPASAD Conferência Pan-Americana de Saúde e Ambiente no Contexto do Desenvolvimento Sustentável 1 Health, environment and development : reflections on the COPASAD experience - Pan-A. Ciências \& Saúde Coletiva, v. 3, n. 2, p. 33-46, 1998.

US EPA, keep out of reach of children danger: precautionary statement hazards to humans and domestic animals. TIMSEN, 1994.

US EPA. Emissions of Organic Air Toxics from Open Burning. United States Environmental Protection Agency, v. EPA-600/R-, October, 2002.

VERAS, M. M. et al. Air pollution and effects on reproductive-system functions globally with particular emphasis on the brazilian population. Journal of Toxicology and Environmental Health - Part B: Critical Reviews, v. 13, n. 1, p. 1-15, 2010.

WALTER, R.; GOTTLIEB, D. J.; O'CONNOR, G. T. Environmental and Genetic Risk Factors and Gene-Environment Interactions in the Pathogenesis of Chronic Obstructive Lung Disease. Environmental Health Perspectives, v. 108, n. s4, p. 733-742, 2000.

WEVERS, M.; DE FRÉ, R.; DESMEDT, M. Effect of backyard burning on dioxin deposition and air concentrations. Chemosphere, v. 54, n. 9, p. 1351-1356, 2004.

WHO - WORLD HEALTH ORGANIZATION - EUROPE. Population health and waste management: scientific data and policy options. Report of a WHO workshop, Rome, Italy 29-30 March 2007. World Health Organization, March, p. 29-30, 2007.

WHO - WORLD HEALTH ORGANIZATION - Children are not little adults. Children's Health and the Environment, 2008.

\section{Como citar este artigo (Formato ABNT):}

BRANDÃO JÚNIOR, Eutrópio Luiz; BERNARDO, Gabriel Pereira; BERNARDO, Lorena Pereira; NASCIMENTO, Sâmia Israele Braz do; LIMA, Bruna Furtado Rolim; SILVA, Kimberly Virginin Cruz Correia da; CVALCANTE, Gabriela Macêdo Egídio; RULIM, Ana Laís Lacerda; DUARTE, Jacob Oliveira. Queima Inadequada de Resíduos Sólidos Domésticos, Principais Gases Tóxicos e Manifestações Clínicas:Uma Revisão de Literatura. Id on Line Rev.Mult. Psic., 2018, vol.12, n.42, p. 602-612. ISSN: 1981-1179.

Recebido: 08/09/2018;

Aceito: 09/10/2018 